\title{
Bose-Einstein condensation of lithium
}

\author{
R. G. Hulet, C. C. Bradley, and C. A. Sackett \\ Physics Department and Rice Quantum Institute, Rice University \\ Houston, Texas 77005-1892 USA
}

\begin{abstract}
Bose-Einstein condensation of ${ }^{7} \mathrm{Li}$ has been studied in a magnetically trapped gas. Because of the effectively attractive interactions between ${ }^{7} \mathrm{Li}$ atoms, many-body quantum theory predicts that the occupation number of the condensate is limited to about 1400 atoms. We observe the condensate number to be limited to a maximum value between 650 and 1300 atoms. The measurements were made using a versatile phase-contrast imaging technique. We discuss our measurements, the current theoretical understanding of BEC in a gas with attractive interactions, and future experiments we hope to perform.
\end{abstract}

Keywords: Bose-Einstein condensation, negative scattering length, lithium, phase contrast imaging

\section{INTRODUCTION}

Bose-Einstein condensation (BEC) has been observed in magnetically trapped atomic gases of ${ }^{87} \mathrm{Rb},{ }^{1}{ }^{23} \mathrm{Na},{ }^{2,3}$ and ${ }^{7} \mathrm{Li}^{4,5}$ Although the condensates are still very dilute gases, the interactions between atoms have been seen to play a significant role in determining their physical properties. For ${ }^{87} \mathrm{Rb}$ and ${ }^{23} \mathrm{Na}$ atoms, the interactions are effectively repulsive, which causes the volume of the condensates to increase and modifies their excitation spectra. ${ }^{3,6,7}$ In contrast, the interactions between ${ }^{7} \mathrm{Li}$ atoms are effectively attractive, and are thought to prevent $\mathrm{BEC}$ from occurring at all in a homogeneous gas. ${ }^{8,9}$ As recently as 1994, attractive interactions were predicted to preclude BEC in a trap as well, ${ }^{9}$ but it is now understood that this is not the case, and that BEC can occur with a limited number of atoms in the condensate. ${ }^{10-16}$ For ${ }^{7} \mathrm{Li}$ in our trap, the predicted limit is about 1400 atoms.

We originally reported evidence for BEC in ${ }^{7} \mathrm{Li}$ in Ref. 4 . In that work, we were not able to observe the condensate directly, so that quantitative analysis was difficult. However, the measurements suggested that the number of condensate atoms $\left(N_{0}\right)$ might be as high as $10^{5}$, which was considerably greater than expected. We now understand the original measurements more completely, and have shown that they were consistent with lower $N_{0} .{ }^{17}$ We have subsequently measured $N_{0}$ more quantitatively, and have obtained values in agreement with the theoretical limit. ${ }^{5}$ In this report, we will discuss our new measurements and their analysis. We will also briefly describe the current theoretical understanding of BEC in a gas with attractive interactions, and some of the experiments we hope to perform in order to further investigate this phenomenon.

\section{THEORY}

Interactions between ultracold atoms may be characterized by a single parameter, the s-wave scattering length $a .^{18}$ The magnitude of $a$ indicates the strength of the interaction, while the sign determines whether the interactions are effectively attractive $(a<0)$ or repulsive $(a>0)$. For densities $n$ such that $n a^{3} \ll 1$, only two-body interactions are important. The scattering length for ${ }^{7} \mathrm{Li}$ is known to be $-1.45 \pm 0.04 \mathrm{~nm},{ }^{19}$ so two-body interactions dominate at the experimentally achieved densities of $n<10^{13} \mathrm{~cm}^{-3}$.

The effects of interactions on the condensate are studied using mean-field theory. ${ }^{18}$ In this approximation, the interaction Hamiltonian is replaced by its mean value, resulting in an interaction energy of $U=4 \pi \hbar^{2} a n / m$, where $m$ is the atomic mass. Because $a<0, U$ decreases with increasing $n$, so the condensate tends to collapse upon itself, making the gas mechanically unstable. In a system with finite volume, however, the zero-point kinetic energy of the atoms provides a stabilizing influence. The net result of these effects can be determined by solving the non-linear Schroedinger equation for the wave function of the condensate, $\psi(r):^{10}$

$$
\left(-\frac{\hbar^{2}}{2 m} \nabla^{2}+V(r)+U(r)-\mu\right) \psi=0 .
$$


Here $\mu$ is the chemical potential, and $V(r)$ is the confining potential provided by the trap. In a spherically-symmetric harmonic trap, $V(r)=m \omega^{2} r^{2} / 2$, for oscillation frequency $\omega$. The interaction energy $U(r)$ is determined by taking $n(r)=N_{0}|\psi(r)|^{2}$. A solution to Eq. (1) is found to exist only when $N_{0}$ is sufficiently small. The limiting $N_{0}$ is approximately equal to $l / a$, where $l$ is the length scale of the condensate, equal to $(\hbar / m \omega)^{1 / 2}$ for a harmonic trap. This limit can be understood physically as requiring that the interaction energy $U$ be small compared to the trap level spacing $\hbar \omega$, so that the interactions act as a small perturbation to the ideal-gas solution. For our trap, the exact limit is 1380 atoms. ${ }^{10}$ At temperature $T>0$, the mean field theory must be modified to take into account the presence of thermally excited atoms, and the limiting $N_{0}$ is somewhat lower. ${ }^{15,16}$ Note that when a solution to Eq. (1) exists, it represents only a metastable state of the trapped atoms, as the equilibrium state of lithium at low temperature is a crystalline metal solid.

For condensate occupation numbers well below the limit, $\psi$ is given by the single-particle ground state of the confining potential. If $N_{0}$ is increased, the interaction energy causes the spatial extent of $\psi$ to decrease. ${ }^{11,13-15}$ At some point before the limit is reached, thermal or quantum-mechanical fluctuations overwhelm the metastability of the state, and the condensate begins to collapse. ${ }^{12,14}$ The collapse is characterized by a rapid increase in the density of the condensate, and correspondingly large loss rates due to inelastic collisions. In principle, the collapse is very complicated, as many-body interactions become important. However, it is very likely that sufficient latent heat is released to eject all the atoms from the trap. The net result of the collapse is therefore to simply eliminate the condensate.

At $T=0$, only condensate atoms can be present, so after the collapse occurs, the trap is empty. However, if $T>0$, then the thermally excited atoms will remain, and the gas will be in a non-equilibrium distribution. As elastic collisions thermalize the gas, $N_{0}$ will begin to grow again. In a closed system the gas will eventually stablize at a chemical potential and temperature such that the equilibrium value of $N_{0}$ is less than the limit. However, if the gas is continuously cooled by some external means, the condensate fill/collapse cycle will repeat until $T=0$ is reached. We are currently attempting to model the dynamics of this system, using the quantum Boltzmann equation. We also wish to experimentally verify as many aspects of this model as possible.

\section{EXPERIMENT}

The apparatus used to produce BEC has been discussed in detail in previous publications. ${ }^{4,5,20}$ The magnetic trap is an Ioffe design, constructed from six cylindrical permanent magnets. The atoms are trapped in the doubly spin polarized ground state, where they experience a nearly symmetric harmonic potential with oscillation frequencies $\nu_{x}=150.6 \mathrm{~Hz}, \nu_{y}=152.6 \mathrm{~Hz}$, and $\nu_{z}=131.5 \mathrm{~Hz}$. $^{*}$ The magnetic field at the center of the trap is directed along the $z$-axis, with a magnitude of $1003 \mathrm{G}$. This field configuration prevents losses due to nonadiabatic spin-flip transitions which can occur near a field zero. ${ }^{21-23}$ The trap is loaded from a laser-slowed atomic beam, and the dissipation needed to capture the atoms is provided by six laser beams tuned near the $2 \mathrm{~S}_{1 / 2}\left(\mathrm{~F}=2, \mathrm{~m}_{F}=2\right) \leftrightarrow 2 \mathrm{P}_{3 / 2}(\mathrm{~F}=3$, $\mathrm{m}_{F}=3$ ) cycling transition. The number of trapped atoms $N$ reaches a maximum of approximately $2 \times 10^{8}$, after a few seconds of loading. These atoms are pre-cooled to about $200 \mu \mathrm{K}$ by Doppler laser cooling, and have a peak density of roughly $1 \times 10^{11} \mathrm{~cm}^{3}$.

After switching off the laser beams, the atoms are further cooled by forced evaporative cooling. ${ }^{24}$ The hottest atoms are driven to an untrapped ground state by a microwave field tuned just above the $\left(\mathrm{F}=2, \mathrm{~m}_{F}=2\right) \leftrightarrow$ $\left(\mathrm{F}=1, \mathrm{~m}_{F}=1\right)$ Zeeman transition frequency of approximately $3450 \mathrm{MHz}$. As the atoms cool, the microwave frequency is reduced. The frequency vs. time trajectory that maximizes the phase-space density of the trapped atoms is calculated ahead of time,${ }^{25}$ and depends on the elastic collision rate and the trap loss rate. Atoms are lost from the trap due to collisions with hot background gas atoms at a rate of approximately $1.6 \times 10^{-3} \mathrm{~s}^{-1}$, and due to inelastic dipolar-relaxation collisions between trapped atoms with a rate constant 0 $10^{-14} \mathrm{~cm}^{3} / \mathrm{s}^{\dagger} .{ }^{27}$ Quantum degeneracy is reached with $N \approx 10^{5}$ atoms at $T \approx 300 \mathrm{nK}$, after roughly 200 seconds of cooling.

After cooling, the trapped atom distribution is observed by imaging via an optical probe. Because a condensate in a trap is spatially localized, this technique can be used to observe BEC directly. ${ }^{28}$ It has also been used to measure the velocity distribution of the gas, by first releasing the atoms from the trap and allowing them to ballistically

\footnotetext{
*Although the trap is constructed from permanent magnets, the values for the oscillation frequencies and bias field have changed from those previously reported. This is because the magnets have been replaced.

tThe rate for three body recombination is expected to be negligible at our densities. ${ }^{26}$
} 


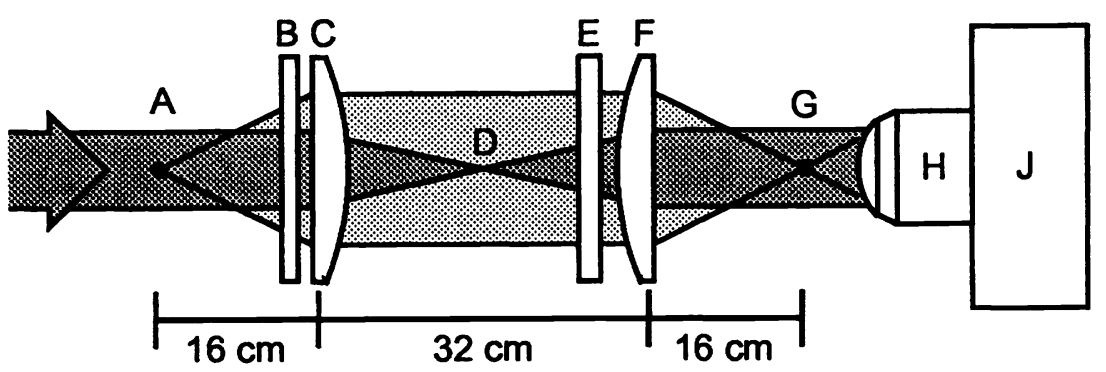

Figure 1. A schematic of the imaging system used for in situ phase-contrast polarization imaging. A linearly polarized laser beam is directed through the cloud of trapped atoms located at $A$. The probe beam and scattered light field pass out of a vacuum viewport $B$, and are relayed to the primary image plane $G$ by an identical pair of 3-cm-diameter, 16-cm-focal-length doublet lenses $\mathrm{C}$ and $\mathrm{F}$. The light is then re-imaged and magnified onto a camera $\mathrm{J}$ by a microscope objective $\mathrm{H}$. The measured magnification is 19 , and the camera pixels are $19 \mu \mathrm{m}$ square. A linear polarizer $\mathbf{E}$ can be used to cause the scattered light and probe fields to interfere, producing an image sensitive to the refractive index of the cloud. The system is focussed by adjusting the position of lens $F$, which is mounted on a translator.

expand. ${ }^{1,2}$ However, because our trap is formed by permanent magnets, the atoms cannot be released and only in situ imaging is possible.

The harmonic oscillator ground state of our trap has a Gaussian density distribution with a $1 / e$-radius of 3 $\mu \mathrm{m}$. The resolution of the imaging system must therefore be sufficient to detect such a small object. In our original experiment, ${ }^{4}$ the imaging resolution was not sufficient, but the presence of the condensate was deduced from distortions observed in images of quantum degenerate clouds. We now have a greater understanding of these distortions, and they are explained more fully in Ref. 17. In that work, we confirm that the presence of a condensate should cause such distortions, given the imaging system which was used.

We have subsequently improved our imaging, and now use a system shown schematically in Fig. 1 . With the polarizer $E$ removed, it can be used to measure the density distribution by absorption imaging, in which the absorptive shadow of the atoms is imaged onto the camera. However, the atom cloud causes both an attenuation and a phase shift of the probe beam. Near resonance, the phase shift can be large, and any imaging system with finite resolution will be sensitive to this phase to some extent. This sensitivity can cause significant image distortions, which are readily detected through their dependence on the probe detuning $\Delta$. In order to eliminate such distortions, it is necessary to reduce the index of refraction by using large $\Delta$. However, since the absorption coefficient decreases as $\Delta^{-2}$, while the index decreases as only $\Delta^{-1}$, eliminating the distortions can leave the absorption signal too small to be detected.

This problem can be solved by using phase-contrast imaging, a common technique in microscopy. ${ }^{29}$ The net effect of the atoms on the incident electric field $\mathbf{E}_{0}$ can be described by a complex phase $\beta$, such that immediately after the cloud the field is $\mathbf{E}=\mathbf{E}_{0} e^{i \beta}$. The phase $\beta(r)$ depends on position, and has real and imaginary parts given by $\beta=\phi+i \alpha / 2$, where $\phi$ is the dispersive phase shift and $\alpha$ is the optical density of the cloud. In perfect absorption imaging, the detected signal intensity $I_{s}$ depends only on $\alpha: I_{s}=I_{0}\left|e^{i \beta}\right|^{2}=I_{0} e^{-\alpha}$, where $I_{0}=\left|\mathbf{E}_{0}\right|^{2}$. Phasecontrast techniques consist of controlled ways to produce an image intensity which depends on $\phi$. In the simplest technique, dark-field imaging, a spatially small opaque beam block is inserted at a focus of the probe laser beam (position D in Fig. 1), thus blocking the probe beam itself but little of the scattered light. The resulting signal is $I_{s}=I_{0}\left|e^{i \beta}-1\right|^{2} \approx I_{0} \phi^{2}$ for $\alpha \ll|\phi| \ll 1$. Andrews et al. used this technique to image ${ }^{23} \mathrm{Na}$ Bose-Einstein condensates in situ. ${ }^{28}$ However, in order to minimize refractive distortions it is desireable that $|\phi| \ll 1$, so the dark-field signal becomes relatively small as it is proportional to $\phi^{2}$. We have implemented a more sensitive technique, in which the signal depends linearly on $\phi$.

The method we use exploits the birefringence of the atoms in a strong magnetic field. The probe beam is linearly polarized perpendicular to the magnetic field axis, and propagates along an axis inclined $55^{\circ}$ with respect to the field. The electric field of the probe decomposes into two elliptical polarizations $\mathbf{E}=\mathbf{E}_{c}+\mathbf{E}_{n c}$, such that $\mathbf{E}_{c}$ couples 
to the $\sigma^{+}$optical transition and acquires a phase-shift, while $\mathbf{E}_{n c}$ does not. By passing the transmitted light through a polarizer ( $E$ in Fig. 1), $\mathbf{E}_{c}$ and $\mathbf{E}_{n c}$ can be coherently recombined. The interference pattern which this produces is a phase-contrast image of the cloud, with the detected signal given by

$$
I_{s}=\frac{I_{0}}{16}\left[\cos ^{2} \theta\left(1+9 e^{-\alpha}+6 e^{-\alpha / 2} \cos \phi\right)+\sin ^{2} \theta\left(3+3 e^{-\alpha}-6 e^{-\alpha / 2} \cos \phi\right)-8 \sqrt{3} \sin \theta \cos \theta e^{-\alpha / 2} \sin \phi\right],
$$

where $\theta$ is the angle between the polarizer axis and the initial polarization of the probe beam. The coefficients in Eq. (2) arise from the angle of incidence of the probe. Usually, the probe detuning is large enough that $\phi$ is small and $\alpha$ can be neglected, so that the signal can be expanded as

$$
I_{s}(r)=I_{0}\left(\cos ^{2} \theta+\frac{\sqrt{3}}{4} \phi(r) \sin 2 \theta-\frac{3}{16} \phi(r)^{2} \cos 2 \theta\right),
$$

Linear phase-contrast imaging is accomplished for $\theta=45^{\circ}$ and dark-field imaging is recovered for $\theta=90^{\circ}$. By varying $\theta$ between these extremes, the relative size of the signal and background can be varied in order to maximize the signal-to-noise ratio of the image. For the data reported here, $\theta= \pm 75^{\circ}$.

The probe beam is pulsed on for a duration of $10 \mu \mathrm{s}$, at an intensity of $250 \mathrm{~mW} / \mathrm{cm}^{2}$ and with $\Delta$ in the range $20 \Gamma<|\Delta|<40 \Gamma$, where $\Gamma=5.9 \mathrm{MHz}$ is the natural linewidth of the transition. Only one image can be obtained because each atom scatters a few photons while being probed, heating the gas to several $\mu \mathrm{K}$.

The detected signal intensity, given by Eq. (3), is related to the density distribution $n$ of the trapped atoms by ${ }^{30}$

$$
\beta\left(x^{\prime}, y^{\prime}\right)=\phi+i \frac{\alpha}{2}=-\frac{\sigma_{0}}{2} \int d z^{\prime} n\left(x^{\prime}, y^{\prime}, z^{\prime}\right) \frac{\Gamma}{2 \Delta+i \Gamma},
$$

where $\sigma_{0}=1.43 \times 10^{-9} \mathrm{~cm}^{2}$ is the resonant light scattering cross section. The $z^{\prime}$-axis is parallel to the probe propagation axis, while the $x^{\prime}$ - and $y^{\prime}$-axes are perpendicular to it. Light scattering might by modified by the quantum degenerate nature of the atoms, but this effect is expected to be negligible under our conditions. ${ }^{31} \mathrm{Eq}$. (4) also assumes that the atom cloud is thin enough that the laser field is not diffracted or refracted appreciably while passing through. This approximation is accurate for sufficiently small $\phi$.

Because the trap is not isotropic, the density distributions are slightly ellipsoidal; the images are observed to have the expected aspect ratio of 1.10 , accounting for the trap asymmetry and the oblique viewing angle ${ }^{\ddagger}$. Fig. 2 shows radial signal profiles, which are obtained from the images by angle-averaging the data around appropriate ellipses. The various curves are the results of our analysis, which will be described in the following section.

\section{IMAGE ANALYSIS}

We assume that the gas is in thermal equilibrium, and fit $T$ and $N_{0}$ to the data. Any two of $N, T$, or $N_{0}$ completely determine the density of the gas through the Bose-Einstein distribution function. Given $T$ and $N_{0}$, the density is calculated using a semi-classical ideal-gas approximation for the non-condensed atoms ${ }^{\S},{ }^{33}$ and a Gaussian function for the condensate. The solid lines in Fig. 2 show the results of the fits.

For temperatures sufficiently greater than the critical temperature $T_{c}$, the gas can be described by the Boltzmann distribution, which predicts a Gaussian density profile. The long-dashed lines in Fig. 2 are Gaussian functions fit to the tails of the distributions, which approximate the data only in Fig. 2(a), where the fit to the Bose-Einstein distribution indicates that $T \approx T_{c}$. Fig. 2(b) shows three distributions for which $T<T_{c}$. For these distributions, the density is distinctly non-Gaussian, due to an enhanced central peak. Comparison between Fig. 2(a) and the upper curve in Fig. 2(b) is striking, as these distributions correspond to nearly the same temperature, and only differ by about $10 \%$ in number. From this comparison, it is clear that the gas has reached the degenerate regime, where Boltzmann statistics are inadequate.

In order to demonstrate the effect of the condensate, the theoretical contribution of the condensate was subtracted from the solid curves. The remaining signal is shown by the short-dashed curves in Fig. 2(b). As comparison of

\footnotetext{
$\$$ The condensate itself is expected to have an aspect ratio of 1.05 , but this difference is not discernable with our imaging resolution.

${ }^{5}$ The semi-classical result was compared to an exact calculation, and found to be accurate, except for a temperature shift as noted in Ref. 32. All the temperatures reported here are calculated in the semi-classical approximation.
} 


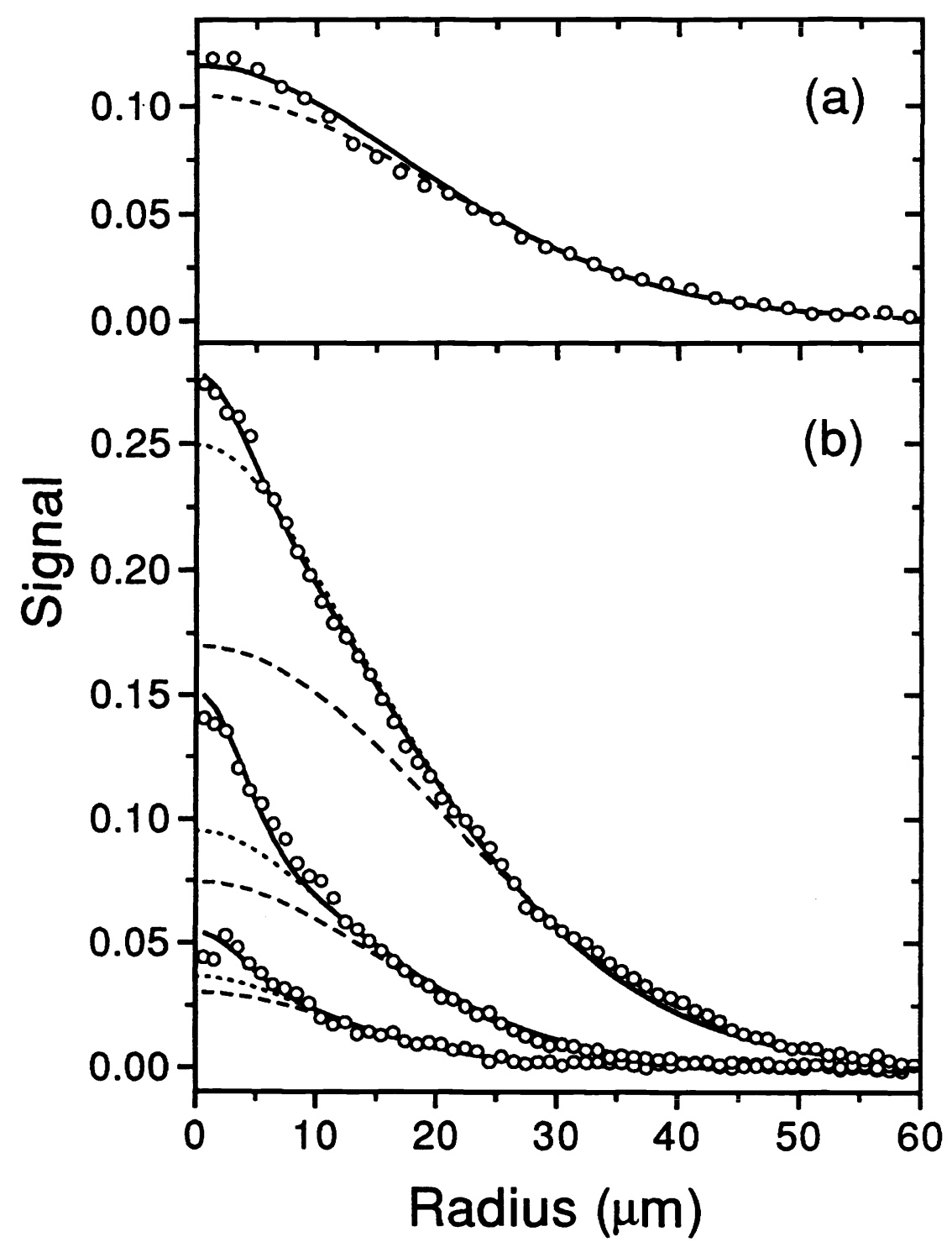

Figure 2. Spatial profiles of trapped ultracold ${ }^{7} \mathrm{Li}$ gas. The vertical axis gives the magnitude of the phase-contrast signal intensity relative to the probe intensity. The data points are taken from observed images. The solid curves are calculated by fitting Bose-Einstein distributions to the data, the short-dashed curves are the same distributions with the condensate atoms removed, and the long-dashed curves are classical (Gaussian) distributions fit to the tails of the data. The calculated signals are convolved with a Gaussian function to account for the limited resolution of the imaging system, assuming an effective resolution of $4 \mu \mathrm{m}$. For the data in (a), the probe detuning was +191 $\mathrm{MHz}$, and the fitted distribution has $9.0 \times 10^{4}$ atoms at a temperature of $309 \mathrm{nK}$. The number of condensate atoms is $\sim 1$, indicating that the gas is just approaching degeneracy. In (b) a sequence of profiles that exhibit condensate peaks are shown. From the strongest to weakest signals, the total number of atoms and fitted temperatures are: $1.01 \times 10^{5}$ atoms at $304 \mathrm{nK} ; 2.6 \times 10^{4}$ atoms at $193 \mathrm{nK}$; and $6.6 \times 10^{3}$ atoms at $122 \mathrm{nK}$. The corresponding numbers of condensate atoms are 500,810 , and 270 , respectively. The probe detuning for these data was $-130 \mathrm{MHz}$. 
the short-dashed, long-dashed and solid curves indicates, the observed increase in the peak signal is caused by both condensed and non-condensed atoms. The contribution of the non-condensed atoms is significant for the upper curve, but at lower temperatures, the contribution of the condensate makes up most of the enhanced peak.

The analysis of the data described above is complicated by the fact that the condensate size is on the order of the imaging resolution. The resolution is included in the fitting procedure by convolving the theoretical signal with the point transfer function of the imaging system, $G(r)$. The value of $G$ at image point $r$ is the field produced at $r$ by a point source at the center of the trap. It is given by the Fourier transform of the phase error of the lenses across the aperture of the imaging system. ${ }^{34}$ The phase error is determined by tracing light rays the through the system, using the known lens composition and geometry, and was found to consist mainly of spherical aberration having a magnitude of $0.5 \lambda / \mathrm{cm}^{4}$.

In order to test the calculation of $G$, we used the imaging system to examine laser light emerging from an optical fiber. The intensity distribution of the light in the fiber is Gaussian with a $1 / e$-radius of $1.2 \mu \mathrm{m}$, so that it approximates a point source. Fig. 3 shows cross-sections of the images obtained with the system focussed at two different points. The narrower peak shown has a $1 / e$-radius of $3.0 \mu \mathrm{m}$, as compared with $2.5 \mu \mathrm{m}$ expected for a diffraction-limited lens (dotted line).

Since the images of the atom cloud are produced by coherently diffracted light, the point transfer function must be convolved with the electric field $E_{0} e^{i \beta}$. The results of the coherent field convolution indicate that the primary effect of the coherence is to reduce the importance of the tail of $G$, because the phase of $G$ in the tail is rapidly varying.

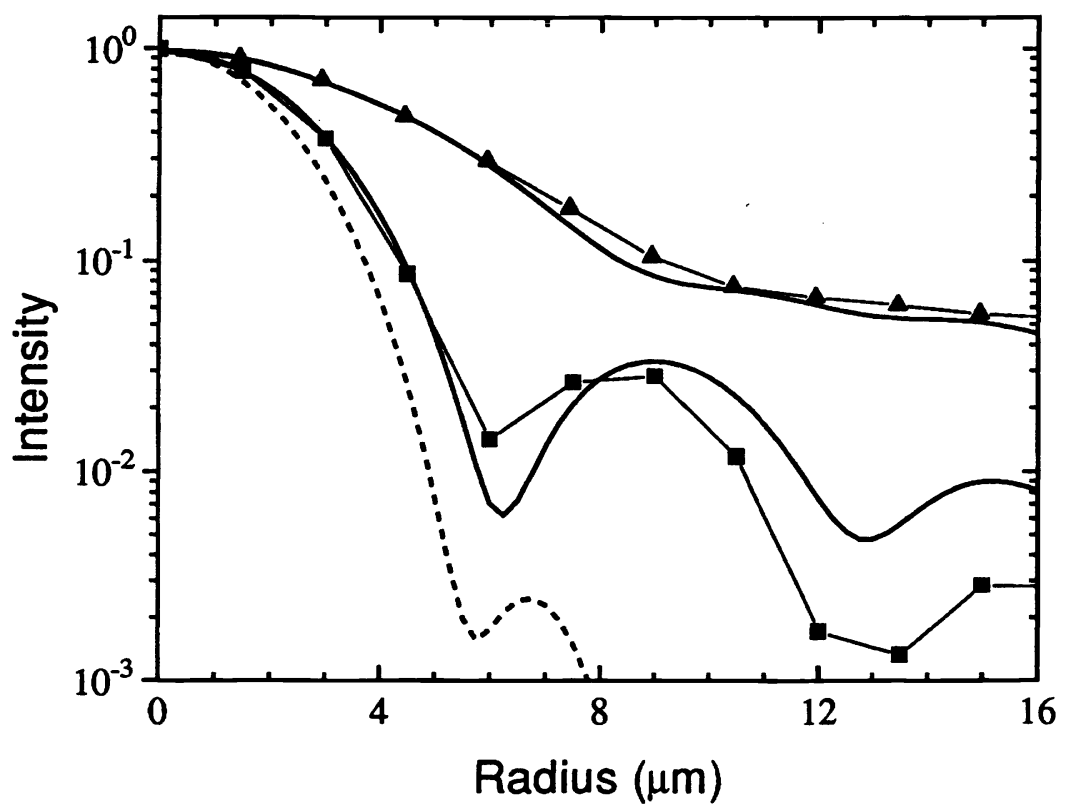

Figure 3. Test images of an optical fiber. The data points are obtained from cross-sections through images of the light emitted by an optical fiber. The squares indicate data obtained with the imaging system at its best focus, and the triangles indicate data obtained with the system defocussed by $200 \mu \mathrm{m}$. The solid curves are the expected intensity patterns, and the dashed curve is the expected pattern for a well-focussed system in the absence of lens aberrations. The curves are obtained by convolving the point transfer function of the with a Gaussian electric field with $1.75 \mu \mathrm{m} 1 / e$-radius to account for the size of the optical fiber mode, and squaring the result to obtain the intensity. The signal is then averaged to reflect the pixel size of the camera. 
In addition, the form of $G$ depends on where the imaging system is focussed, as Fig. 3 shows. When imaging the atom cloud, the focal position can only be determined to $\pm 200 \mu \mathrm{m}$, by observing image distortions which occur when the system is further off focus. Given this uncertainty, and the unimportance of the tail, the imaging convolution is well-approximated by an incoherent convolution of the signal intensity with a Gaussian function of appropriate $1 / e$-radius $R$. As this procedure is computationally simpler and conceptually clearer, we use it here. The value of $R$ can be considered the effective resolution of the imaging system, and across the experimental range of focal positions it ranges from $2.5 \mu \mathrm{m}$ to $5 \mu \mathrm{m}$. This uncertainty in the effective resolution is the dominant source of error in our determination of $N_{0}$.

We have observed degenerate conditions for $T$ between 120 and $330 \mathrm{nK}$, and for $N$ between 6,800 and 135,000 atoms. In all cases, $N_{0}$ is found to be relatively small. Fitting with $R=5 \mu \mathrm{m}$, the maximum $N_{0}$ observed is about 1300 atoms. This value drops to 1000 for $R=4 \mu \mathrm{m}$, and to 650 for $R=2.5 \mu \mathrm{m}$. No systematic effects were observed as either the sign or magnitude of the detuning or the polarizer angle were varied, indicating the absence of phase-dependent distortions and confirming the relations given in Eqs. (3) and (4).

In the analysis so far we have assumed that the gas is ideal, but interactions are expected to alter the size and shape of the density distribution. Mean-field theory predicts that interactions will reduce the $1 / e$-radius of the condensate from $3 \mu \mathrm{m}$ for low occupation number to $\sim 2 \mu \mathrm{m}$ as the maximum $N_{0}$ is approached. ${ }^{11,13-16}$ If the smaller condensate radius is used in the fit, the maximum values for $N_{0}$ decrease, becoming $\sim 1050$ for $R=5 \mu \mathrm{m}$. The size of the condensate is not expected to change appreciably for $N_{0}<1000$, so the values obtained for $R=2.5 \mu \mathrm{m}$ and $4 \mu \mathrm{m}$ are not sensitive to interactions. Interactions are not expected to significantly affect the distribution of the non-condensed atoms, ${ }^{15,35}$ because at the critical density the mean interaction energy of $\sim 1 \mathrm{nK}$ is much smaller than $T$.

An estimate of our sensitivity to condensate atoms can be obtained from the fitting procedure. By fixing $N_{0}$ and fitting $T$ to the data, $\chi^{2}$ can be determined as a function of $N_{0}$. Since $N_{0}$ mostly affects the central part of the distribution, we define a restricted, unnormalized $\chi^{2}$ by summing over the squares of the differences between the calculated distributions and the data for radii less than $10 \mu \mathrm{m}$. Calculating $\chi^{2}\left(N_{0}\right)$ for several images with large values of $N_{0}$ indicates that $\chi^{2}$ is increased by a factor of 2 from its minimum value when $N_{0}$ is varied by about 150 atoms, roughly independent of $R$. The ratio of the measured $N_{0}$ to this value serves as an estimate for the robustness of the measurement.

\section{CONCLUSIONS AND FUTURE EXPERIMENTS}

We believe that our measurements have clearly demonstrated that BEC can occur in a gas with $a<0$. The results of our our analysis indicate a number of condensate atoms which is consistent with the limit predicted by mean-field theory, and the range of numbers and temperatures across which the limit is observed to hold suggests that the limit is fundamental, rather than technical. However, we anticipate several further experiments which should provide a greater understanding of this phenomenon.

As previously stated, the dominant uncertainty in our determination of $N_{0}$ is the sensitivity to the effective resolution $R$. This sensitivity could be reduced by observing a distribution consisting mainly of condensate atoms. Because the integrated intensity of an image is independent of lens aberrations, $N_{0}$ could be measured by simply integrating the signal to determine the number of trapped atoms, It may be possible to produce such a $T=0$ distribution using evaporative cooling, but doing is technically difficult. Fluctuations of the trap bias field must be less than about $50 \mu \mathrm{G}$ so that the thermally excited atoms may be removed while leaving the condensate intact. Fluctuations in the trap field arise from variation in the ambient magnetic field and variation of the temperature of the trap magnets, so both of these variables must be controlled precisely.

If evaporative cooling can be repeated reliably and $N_{0}$ measured precisely, the collapse/fill model described in $\S 2$ predicts that the values of $N_{0}$ observed should fluctuate randomly between zero and the limiting value. Observation of these fluctuations should provide information on the nature of the collapse and fill processes, as well as confirming the model. Fluctuations in $N_{0}$ have been observed already, but it is not yet clear whether they are intrinsic or whether they are caused by variations in trap loading and evaporative cooling.

Finally, it may be possible to modify the scattering length by applying a laser beam tuned near a molecular resonance of $\mathrm{Li}_{2} \cdot{ }^{36,37}$ While it should be possible to make $a$ positive using this method, doing so would require a high 
laser intensity. In the presence of such a bright laser, the lifetime of atoms in the trap is reduced due to Rayleigh scattering. Because evaporative cooling is slow, it would not be possible to populate the condensate with a significant number of atoms in the available time. However, it should be possible to make $a$ more negative, and thereby induce a collapse. By controlling when the collapse occurs, this technique would allow direct investigation of the dynamics of the collapse.

\section{ACKNOWLEDGEMENTS}

We are grateful for helpful discussions with T. Bergeman, W. Ketterle, H. Stoof, E. Timmermans, N. Vansteenkiste, M. Welling, and C. Westbrook. This work is supported by the National Science Foundation and the Welch Foundation.

\section{REFERENCES}

1. M. H. Anderson, J. R. Ensher, M. R. Matthews, C. E. Wieman, and E. A. Cornell, "Observation of Bose-Einstein condensation in a dilute atomic vapor," Science 269, p. 198, 1995.

2. K. B. Davis, M.-O. Mewes, M. R. Andrews, N. J. van Druten, D. S. Durfee, D. M. Kurn, and W. Ketterle, "Bose-Einstein condensation in a gas of sodium atoms," Phys. Rev. Lett. 75, p. 3969, 1995.

3. M.-O. Mewes, M. R. Andrews, N. J. van Druten, D. M. Kurn, D. S. Durfee, and W. Ketterle, "Bose-Einstein condensation in a tightly confining dc magnetic trap," Phys. Rev. Lett. 77, p. 416, 1996.

4. C. C. Bradley, C. A. Sackett, J. J. Tollett, and R. G. Hulet, "Evidence of Bose-Einstein condensation in an atomic gas with attractive interactions," Phys. Rev. Lett. 75, p. 1687, 1995.

5. C. C. Bradley, C. A. Sackett, and R. G. Hulet, "Bose-Einstein condensation of lithium: Observation of limited condensate number," Phys. Rev. Lett., in press.

6. M.-O. Mewes, M. R. Andrews, N. J. van Druten, D. M. Kurn, D. S. Durfee, C. G. Townsend, and W. Ketterle, "Collective excitations of a Bose-Einstein condensate in a magnetic trap," Phys. Rev. Lett. 77, p. 988, 1996.

7. D. S. Jin, J. R. Ensher, M. R. Matthews, C. E. Wieman, and E. A. Cornell, "Collective excitations of a Bose-Einstein condensate in a dilute gas," Phys. Rev. Lett. 77, p. 420, 1996.

8. L. D. Landau and E. M. Lifshitz, Statistical Physics, Pergamon, London, 1st ed., 1958.

9. H. T. C. Stoof, "Atomic Bose gas with a negative scattering length," Phys. Rev. A 49, p. 3824, 1994.

10. P. A. Ruprecht, M. J. Holland, K. Burnett, and M. Edwards, "Time-dependent solution of the nonlinear Schrodinger equation for Bose-condensed trapped neutral atoms," Phys. Rev. A 51, p. 4704, 1995.

11. F. Dalfovo and S. Stringari, "Bosons in anisotropic traps: Ground state and vortices," Phys. Rev. A 53, p. 2477, 1996.

12. Y. Kagan, G. V. Shlyapnikov, and J. T. M. Walraven, "Bose-Einstein condensation in trapped atomic gases," Phys. Rev. Lett. 76, p. 2670, 1996.

13. R. J. Dodd, M. Edwards, C. J. Williams, C. W. Clark, M. J. Holland, P. A. Ruprecht, and K. Burnett, "Role of attractive interactions on Bose-Einstein condensation," Phys. Rev. A 54, p. 661, 1996.

14. H. T. C. Stoof, "Macroscopic quantum tunneling of a Bose condensate," J. of Stat. Phys., in press.

15. M. Houbiers and H. T. C. Stoof, "Stability of Bose condensed atomic ${ }^{7}$ Li," Phys. Rev. A 54, p. 5055, 1996.

16. T. Bergeman, to be published.

17. C. C. Bradley, C. A. Sackett, and R. G. Hulet, "Analysis of in situ images of Bose-Einstein condensates of lithium," to be published.

18. K. Huang, Statistical Mechanics, John Wiley \& Sons, New York, 2 ed., 1987.

19. E. R. I. Abraham, W. I. McAlexander, C. A. Sackett, and R. G. Hulet, "Spectroscopic determination of the s-wave scattering length of lithium," Phys. Rev. Lett. 74, p. 1315, 1995.

20. J. J. Tollett, C. C. Bradley, C. A. Sackett, and R. G. Hulet, "Permanent magnet trap for cold atoms," Phys. Rev. A 51, p. R22, 1995.

21. A. L. Migdall, J. V. Prodan, W. D. Phillips, T. H. Bergeman, and H. J. Metcalf, "First observation of magnetically trapped neutral atoms," Phys. Rev. Lett. 54, p. 2596, 1985.

22. W. Petrich, M. H. Anderson, R. J. Ensher, and E. A. Cornell, "Stable, tightly confining magnetic trap for evaporative cooling of neutral atoms," Phys. Rev. Lett. 74, p. 3352, 1995. 
23. K. B. Davis, M.-O. Mewes, M. A. Joffe, M. R. Andrews, and W. Ketterle, "Evaporative cooling of sodium atoms," Phys. Rev. Lett. 74, p. 5202, 1995.

24. W. Ketterle and N. J. van Druten, "Evaporative cooling of trapped atoms", in Advances in Atomic, Molecular, and Optical Physics, B. Bederson and H. Walther, eds., vol. 37, p. 181, Academic Press, San Diego, 1996.

25. C. A. Sackett, C. C. Bradley, and R. G. Hulet, to be published.

26. A. J. Moerdijk, H. M. J. M. Boesten, and B. J. Verhaar, "Decay of trapped ultracold alkali atoms by recombination," Phys. Rev. A 53, p. 916, 1996.

27. A. J. Moerdijk and B. J. Verhaar, "Collisional two- and three-body decay rates of dilute quantum gases at ultralow temperatures," Phys. Rev. A 53, p. R19, 1996.

28. M. R. Andrews, M.-O. Mewes, N. J. van Druten, D. S. Durfee, D. M. Kurn, and W. Ketterle, "Direct, nondestructive observation of a Bose condensate," Science 273, p. 84, 1996.

29. E. Hecht, Optics, Addison-Wesley, Reading, MA, 2nd ed., 1987.

30. P. Meystre and M. Sargent III, Elements of Quantum Optics, Springer-Verlag, Berlin, 2 ed., 1991.

31. O. Morice, Y. Castin, and J. Dalibard, "Refractive index of a dilute Bose gas," Phys. Rev. A 51, p. 3896, 1995.

32. W. Ketterle and N. J. van Druten, "Bose-Einstein condensation of a finite number of particles trapped in one or three dimensions," Phys. Rev. A 54, p. 656, 1996.

33. V. Bagnato, D. E. Pritchard, and D. Kleppner, "Bose-Einstein condensation in an external potential," Phys. Rev. A 35, p. 4354, 1987.

34. M. Born and E. Wolf, Principles of Optics, Pergamon Press, New York, 1959.

35. T. Bergeman, personal communication.

36. P. O. Fedichev, Y. Kagan, G. V. Shlyapnikov, and J. T. M. Walraven, to be published.

37. R. Napolitano, J. Weiner, and P. S. Julienne, personal communication. 